\title{
Recent Epidemiological Trend of Dengue in A Teriary Care Hospital in Kolkata
}

\author{
Dr. Sanjeev Das ${ }^{1}$ Dr.Maitreyi Bandyopadhyay ${ }^{2}$ Dr. Simit Kumar ${ }^{3}$ Dr. Reena \\ Roy Ghosh ${ }^{4}$ Dr. Purnima Mandol ${ }^{5}$ Prof. Manas Bandyopadhayay ${ }^{6}$ Prof.. Mitali \\ Chatterjee
}

\begin{abstract}
Dengue virus infection has emerged as notable public health problem in recent decades because of high mortality and morbidity associated with it. Dengue with its two severe clinical manifestations dengue hemorrhagic fever (DHF) and dengue shock syndrome (DSS) is endemic in India and epidemics are frequently reported from many parts of India and abroad. Dengue fever is a recurrent problem in West Bengal. The present study had concentrated on the epidemiology and trend of Dengue fever in a tertiary care hospital of Kolkata Blood was collected aseptically from 7141 clinically suspected patients over a period of four years ,attending the hospital \& suffering from fever for at least five days \& serum was separated and analyzed by IgM capture ELISA technique to detect Dengue specific IgM antibodies. 789 out of 7141 samples were found to be reactive (11.04\%) and total of 493 cases were male (62.48\%) whereas 296 cases were female $(37.52 \%)$. Majority of the cases (46.9\%) were in the age group 10 to 29 years. A clear post rainy season peak was noted in each year during the study period. There was a rise of number of Dengue cases in 2012, then a fall in 2013 and again a sharp peak was noted in 2015.Dengue IgM sensitivity among the suspected cases indicates active dengue virus activity. Further Studies like Haemagglutination inhibition test need to be done to identify circulating serotypes of dengue virus to design preventive strategies
\end{abstract}

Keywords: Dengue, Epidemiology, IgM capture ELISA,

\section{Introduction}

Dengue virus is a mosquito-borne flavivirus and one of the most prevalent arbovirus in tropical and subtropical regions of the world. Dengue virus infection has emerged as notable public health problem in recent decades especially in the tropical and subtropical countries because of high mortality and morbidity associated with it ${ }^{1}$. Dengue with its two severe clinical manifestations dengue hemorrhagic fever (DHF) and dengue shock syndrome (DSS) is endemic in India and epidemics are frequently reported from many parts of India and abroad. Dengue fever is a recurrent problem in West Bengal. In Kolkata (Calcutta) Dengue was first documented in 1824. Thereafter several epidemics of Dengue were observed in Kolkata in 1836, 1906, 1911 and 1923 with a severe epidemic in 1923 affecting about 40\% of people. In India, DHF was first reported in Kolkata in 1963-64 where 200 people died. Another small outbreak of DHF in Kolkata occurred in 1990 where 12 children died. Recently, there has been a large outbreak in 2005 involving all districts of West Bengal with 6293 cases and 27 deaths. In Kolkata alone 3967 persons were affected with 14 deaths ${ }^{2}$, this has been followed by smaller number of cases occurring in subsequent years. With increasing global temperature and associated climatic changes there are apprehensions about an outbreak in coming years.

Dengue virus is a positive-stranded encapsulated RNA virus. The genomic RNA is approximately 11 $\mathrm{kb}$ in length and is composed of three structural protein genes that encode the nucleocapsid or core protein $(\mathrm{C}), \mathrm{a}$ membrane associated protein (M), an envelope protein (E), and seven nonstructural (NS) protein genes ${ }^{2}$

There are four distinct serotypes, serotypes 1 to 4 . Infection induces a life-long protective immunity to the homologous serotype but confers only partial and transient protection against subsequent infections by the other three serotypes. Instead, it has generally been accepted that secondary infection or infection with secondary or multiple infections with various dengue virus serotypes is a major risk factor for DHF and DSS due to antibodydependent enhancement. ${ }^{4}$

Complications are more common in children. Sequential infections by different serotypes have been implicated for contributing to complications especially if infection with DV-1 is followed by DV-2 or DV $-3^{5}$.

Laboratory diagnosis of dengue virus infection can be made by the detection of specific virus, viral antigen, genomic sequence, and/or antibodies. After the onset of illness, the virus is found in serum or plasma, circulating blood cells, and selected tissues, especially those of the immune system, for approximately 2 to 7 days, roughly corresponding to the period of fever .

Traditionally, Dengue is diagnosed on basis of clinical criteria supported by serological report. Serology is classically positive from 5 days fever onwards. ${ }^{6}$ This leaves a window period where Dengue can be diagnosed and patient can be monitored more effectively. 
The acquired immune response following a dengue infection consists of the production of $\operatorname{IgM}$ and $\operatorname{IgG}$ antibodies primarily directed against the virus envelope proteins. The immune response varies depending on whether the individual has a primary (first dengue or other flavivirus infection) versus a secondary (had dengue or other flavivirus infection in past) dengue infection.

A primary dengue infection is characterized by a slow and low titer antibody response. IgM antibody is the first immunoglobulin isotype to appear. Anti-dengue IgG is detectable at low titer at the end of the first week of illness, and slowly increases. In contrast, during a secondary infection, antibody titers rise extremely rapidly and antibody reacts broadly with many flaviviruses. High levels of IgG are detectable even in the acute phase and they rise dramatically over the preceding two weeks.

IgM capture ELISA (MAC-ELISA) has become an important tool for routine dengue diagnosis, MACELISA has a sensitivity and specificity of approximately $90 \%$ and $98 \%$, respectively but only when used five or more days after onset of fever.

\section{Objectives}

1. To find out the Seroprevalence of Dengue among the symptomatic patients attending a tertiary care hospital in Kolkata.

2. Documentation of the present scenario and recent trends of Dengue among the symptomatic patients attending the same hospital in Kolkata.

\section{Materials \& Methods}

The study was performed in the department of Microbiology R G Kar Medical College \& Hospital, Kolkata over a period of four years (From January 2012 to December 2015) with six hundred and seventy three clinically suspected patients attending the hospital \& suffering from fever for at least five days. Blood was collected aseptically from suspected cases, serum was separated and analyzed by $\operatorname{IgM}$ capture ELISA technique to detect Dengue specific IgM antibodies.

Inclusion criteria were as follows:

Fever with two or more of the following symptoms- rash, bodyache, malaise, myalgia, arthralgia and retroorbital pain and eye congestion.

Known immunocompromised patients were excluded from the study.

\section{Results}

A total of 7141 suspected cases were tested by Dengue IgM Capture ELISA over four years and 789 samples were found to be reactive (11.04\%). A total of 493 cases were male $(62.48 \%)$ where as 296 cases were female $(37.52 \%)$. Male predominance is observed over female cases throughout the study period Majority of the cases (46.9\%) were in the age group 10 to 29 years in last four years. A clear post rainy season peak was noted in each year during the study period.

There was a peak observed in 2012, then a fall in 2013 and again a sharp peak was noted in 2015

Figure-1: Gender distribution of Dengue cases

\begin{tabular}{|l|l|l|l|l|}
\hline & 2012 & 2013 & 2014 & 2015 \\
\hline M & 315 & 8 & 28 & 142 \\
\hline F & 210 & 5 & 19 & 62 \\
\hline
\end{tabular}

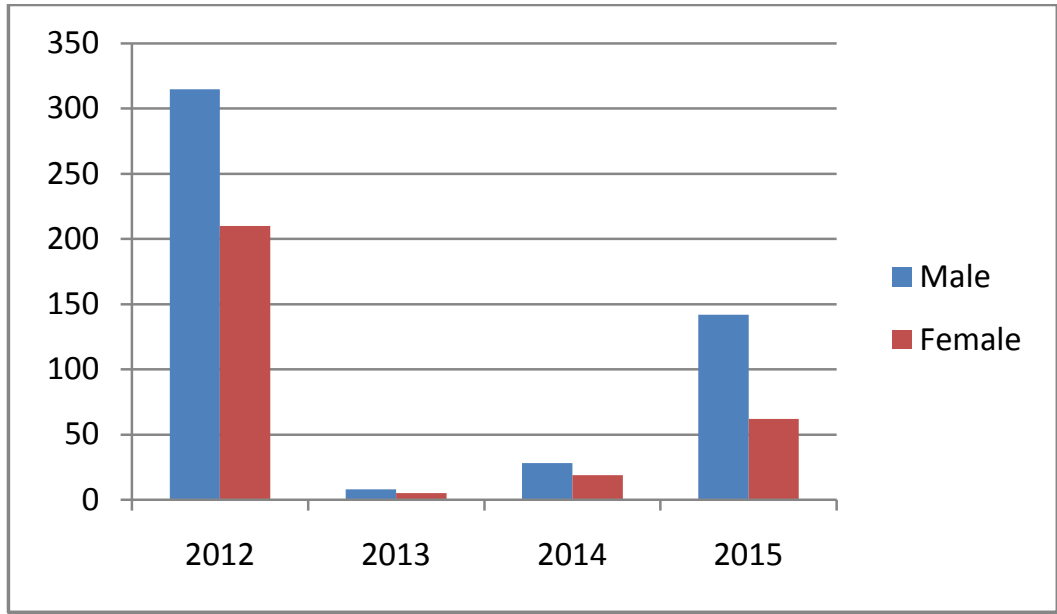




\begin{tabular}{|l|l|l|l|l|}
\hline & 2012 & 2013 & 2014 & 2015 \\
\hline$<10$ years & 99 & 1 & 8 & 47 \\
\hline $10-29$ years & 242 & 7 & 23 & 98 \\
\hline 30-49 years & 147 & 4 & 14 & 46 \\
\hline$>50$ years & 37 & 1 & 2 & 13 \\
\hline
\end{tabular}

Figure-2: Age wise distribution of Dengue cases

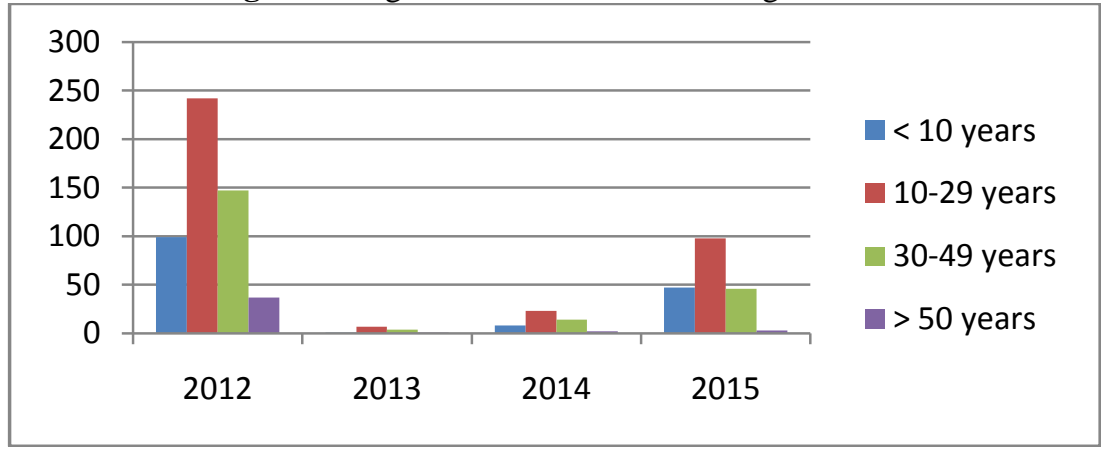

\begin{tabular}{|l|l|l|l|l|}
\hline & $\mathbf{2 0 1 2}$ & $\mathbf{2 0 1 3}$ & $\mathbf{2 0 1 4}$ & $\mathbf{2 0 1 5}$ \\
\hline JANUARY & 0 & 0 & 0 & 4 \\
\hline FEBRUARY & 0 & 0 & 0 & 1 \\
\hline MARCH & 0 & 0 & 0 & 2 \\
\hline APRIL & 1 & 0 & 0 & 0 \\
\hline MAY & 1 & 0 & 0 & 5 \\
\hline JUNE & 2 & 0 & 1 & 2 \\
\hline JULY & 0 & 1 & 0 & 1 \\
\hline AUGUST & 11 & 3 & 0 & 2 \\
\hline SEPTEMBER & 198 & 3 & 8 & 18 \\
\hline OCTOBER & 208 & 2 & 14 & 50 \\
\hline NOVEMBER & 34 & 3 & 21 & 66 \\
\hline DECEMBER & 70 & 1 & 3 & 53 \\
\hline
\end{tabular}

Figure-3: Seasonal distribution of Dengue cases

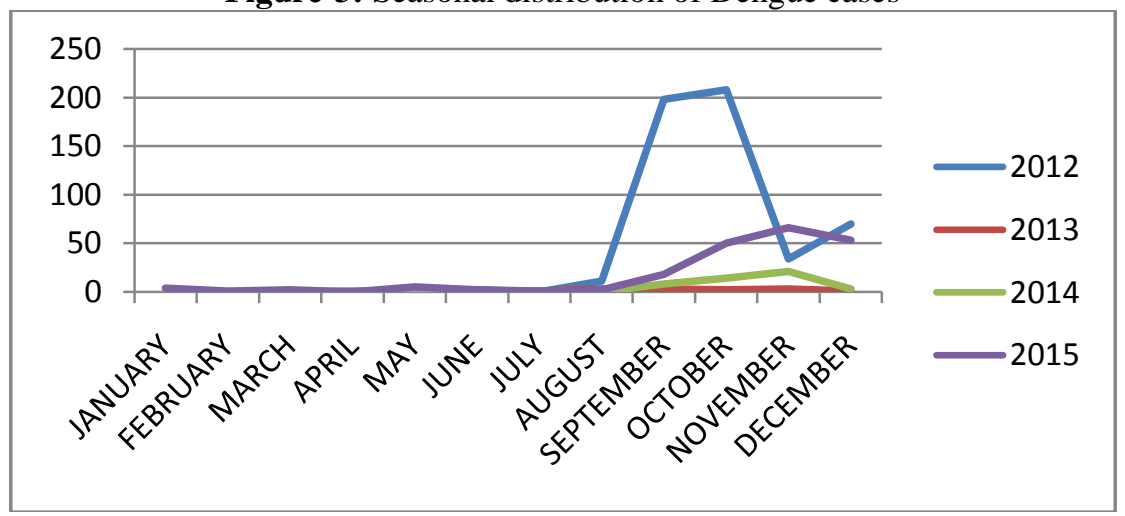

Figure-4: Year wise distribution of Dengue cases.

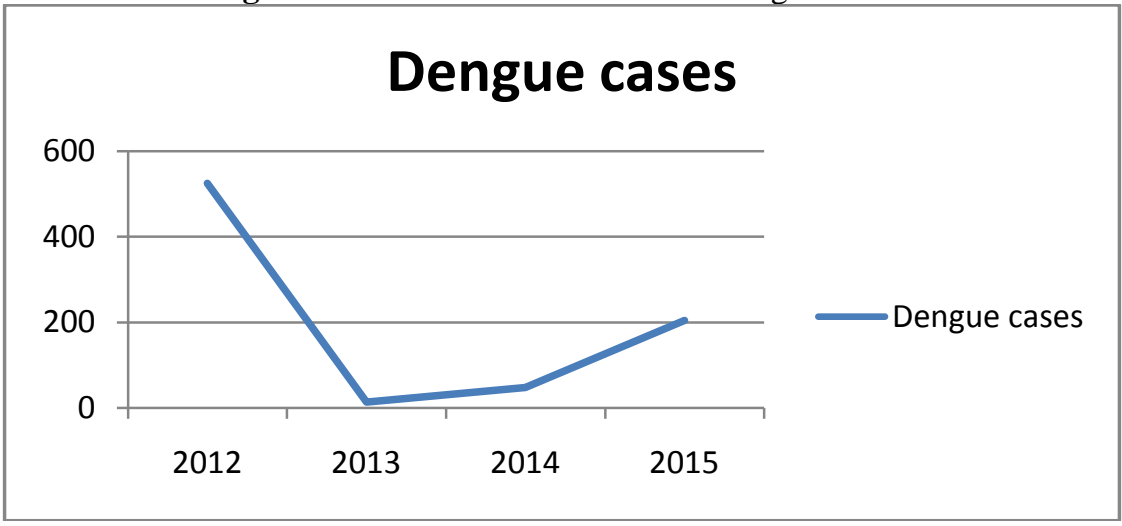




\section{Discussion}

The present study had concentrated on the epidemiology and trend of Dengue fever in a tertiary care hospital of Kolkata since 2012.

In this study, the highest numbers of cases (46.9\%) were recorded in the age group 10-29 yrs with male preponderance. Findings of Gupta et $\mathrm{al}^{7}$. and Chakravarti and Kumaria ${ }^{8}$ were similar to our study i.e, maximum cases in the age group 21-30 years and male patients clearly outnumbered female patients. However Sarkar et al. ${ }^{9}$, reported that maximum cases were in the age group $\leq 10$ years and there was female preponderance.

The majority of the cases were reported during the post monsoon seasons (August to November) with a peak during September and October in all the past four years (2012-2015), in accordance with the established findings by P. Reiter ${ }^{10}$.

Our study has revealed the fact that there was a dengue outbreak in West Bengal in 2012 and 2015. Our finding in this present study corroborates with the findings of Hati ${ }^{11}$, Taraphdar et al. ${ }^{12}$, and Sarkar et al ${ }^{13}$.

\section{Summary \& Conclusion}

The present study had concentrated on the epidemiology and trend of Dengue fever in a tertiary care hospital of Kolkata since 2012.

The study was performed in the department of Microbiology, R G Kar Medical College \& Hospital, Kolkata over a period of four years (From January 2012 to December 2015) with Seven thousand one hundred $\&$ forty one clinically suspected patients attending the hospital \& suffering from fever for at least five days. Blood was collected aseptically from suspected cases, serum was separated and analyzed by IgM capture ELISA technique to detect Dengue specific IgM antibodies. Known immunocompromised cases were excluded from the study.Out of total 7141 suspected cases fulfilling the inclusion criteria, 789 samples were found to be reactive $(11.04 \%)$ and total of 493 cases were male $(62.48 \%)$ whereas 296 cases were female $(37.52 \%)$. Thus male predominance is observed over female cases throughout the study period. Majority of the cases $(46.9 \%)$ were in the age group 10 to 29 years in last four years. A clear post rainy season peak was noted in each year during the study period. There was a rise of number of Dengue cases in 2012, then a fall in 2013 and again a sharp peak was noted in 2015. These two Dengue outbreaks in 2012 and 2015 attended the peak in post rainy season months.

Limitations of the study: Haemagglutination inhibition test or Complement Fixation test of preserved Dengue IgM reactive sera are to be performed for Dengue serotyping. This will help to identify the circulating Dengue serotypes to predict complications like Dengue Hemorrhagic Fever (DHF) and Dengue Shock Syndrome(DSS).

\section{References}

[1]. Ukey PM, Bondade SA, Paunipagar PV, Powar RM, Akulwar SL. Study of Seroprevalence of Dengue Fever in Central India .Indian J Community Med -2010;35(4): 517-9.

[2]. Hati AK. Studies on dengue and dengue haemorrhagic fever (DHF) in West Bengal State, India._J Commun Dis. 2006 Mar;38 (2):124-9.

[3]. Leyssen, P., E. D. Clercq, and J. Neyts. 2000. Perspectives for the treatment of infections with Flaviviridae. Clin. Microbiol. Rev. $13: 67-82$

[4]. Halstead, S. B. 1988. Pathogenesis of dengue: challenge to molecular biology.Science 239:476-481.

[5]. Mourya DT, Yadav P. Vector biology of dengue and chikungunya viruses. Indian J Med Res 2006; 124: 475-80.

[6]. World Health Organization. 1997. Dengue haemorrhagic fever: diagnosis, treatment, prevention and control, 2nd ed. World Health Organization, Geneva, Switzerland

[7]. E. Gupta, L. Dar, G. Kapoor, and S. Broor, "The changing epidemiology of dengue in Delhi, India," Virology Journal, vol. 3, article 92, 2006.

[8]. Chakravarti and R. Kumaria, "Eco-epidemiological analysis of dengue infection during an outbreak of dengue fever, India," Virology Journal, vol. 2, article 32, 2005.

[9]. Sarkar, D. Taraphdar, and S. Chatterjee, "Molecular typing of dengue virus circulating in Kolkata, India in 2010," Journal of Tropical Medicine, vol. 2012, Article ID 960329, 5 pages, 2012.

[10]. P. Reiter, "Climate change and mosquito-borne disease," Environmental Health Perspectives, vol. 109, supplement 1, pp. 141$161,2001$.

[11]. K. Hati, "Dengue serosurveillance in Kolkata, facing an epidemic in West Bengal, India," Journal of Vector Borne Diseases, vol. 46, no. 3, pp. 197-204, 2009

[12]. D. Taraphdar, A. Sarkar, M. K. Bhattacharya, and S. Chatterjee, "Sero diagnosis of dengue activity in an unknown febrile outbreak at the Siliguri Town, District Darjeeling, West Bengal," Asian Pacific Journal of Tropical Medicine, vol. 3, no. 5, pp. 364-366, 2010 .

[13]. Sarkar, D. Taraphdar, and S. Chatterjee, "Investigations of recurrent outbreaks of unknown fever, establish rural dengue activity in West Midnapore, a costal district in West Bengal, India," Archives of Clinical Microbiology, vol. 1, no. 4, 2010. 